\title{
Oxygen reduction in alkaline solution at glassy carbon surfaces and the role of adsorbed intermediates
}

\author{
Haozhi Zhang ${ }^{1}$, Chuhong Lin ${ }^{1}$, Lior Sepunaru', Christopher Batchelor-McAuley ${ }^{1}$, Richard G. \\ Compton ${ }^{1}$ \\ ${ }^{1}$ Department of Chemistry, Physical and Theoretical Chemistry Laboratory, University of Oxford, South Parks Road, \\ Oxford OX1 3QZ, United Kingdom
}

\begin{abstract}
Oxygen reduction at glassy carbon (GC) exhibits distinctively different voltammetric behaviour at high $(>10)$ and low $(<10) \mathrm{pH}$. The peak potential is found to be around $-0.4 \mathrm{~V}$ at $\mathrm{pH} 13$, compared to $-0.6 \mathrm{~V}$ at $\mathrm{pH} 7.4$ as measured against a saturated calomel reference electrode. Using experimental voltammetry and numerical simulation, the difference in peak potential is interpreted in terms of a difference in reaction mechanism. At low $\mathrm{pH}, \mathrm{O}_{2}$ reduction is evidenced to proceed via a solution phase pathway initially resulting in the reduction of oxygen to superoxide. Conversely, at higher $\mathrm{pH}$, a different mechanism is favoured involving the formation of a surface bound superoxide species. The switch between the two mechanisms is related to the protonation of the surface bound intermediate under less basic conditions.
\end{abstract}

\section{KEYWORDS}

Electrochemistry, Oxygen reduction, Electron Transfer Kinetics, Carbon surfaces, Adsorbed intermediated

Address correspondence to Richard G. Compton, Richard.compton@chem.ox.ac.uk 


\section{Introduction}

The voltammetric reduction of oxygen on a glassy carbon electrode is unusual in that the reduction is found to occur at a lower potential under more alkaline conditions. [1-3] For example at $\mathrm{pH} 13$ the peak potential is ca. $-0.40 \mathrm{~V}$, whereas at $\mathrm{pH} 7.4$ it is ca. $-0.60 \mathrm{~V}$. This anodic shift in the position of the voltammetric wave is the opposite of that predicted thermodynamically on the basis of the Nernst equation. Due to the occurrence of coupled protonation steps during the course of the reduction the reaction is overall less energetically favorable at higher $\mathrm{pH}$. [4,5] Consequently, if the reduction were reversible, the peak potential - in contrast to experiment - would be predicted to occur at a more cathodic (negative) potential under alkaline conditions. This unusual voltammetric behavior was recognized and reported as early as the 1970s by Taylor and Humphray [1,2]; However, it took until the 1990s and the work of McCreery [6,7] for the physical origin of this altered behavior to be illuminated.
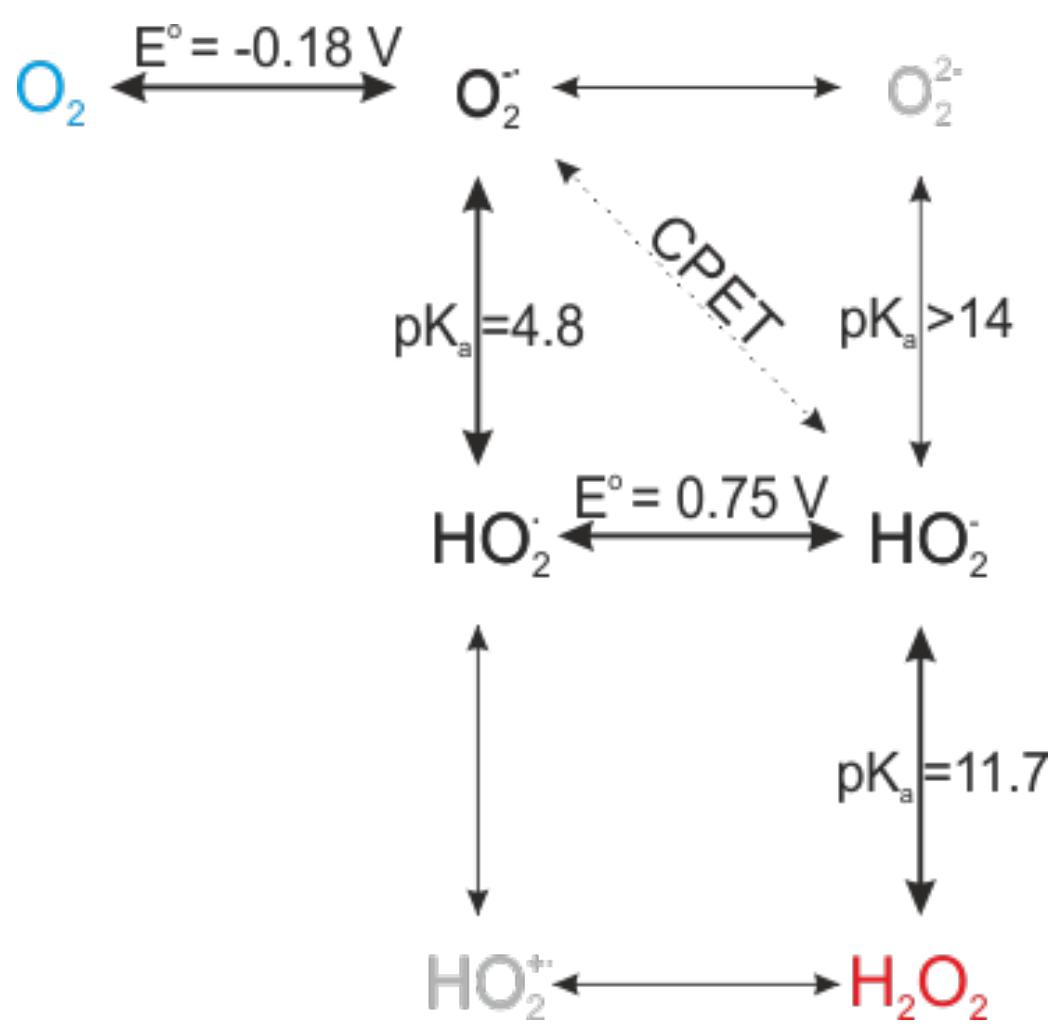

Scheme I: Scheme-of-squares for the oxygen/hydrogen peroxide redox couple. Note potential are reported taking the standard state for all species as unit activity. Dashed diagonal represents a possible concerted-proton-electron transfer (CPET) process. $p K_{a}$ values taken from [8] and standard potentials (vs SHE) calculated from data given in [9]. 
The oxygen reduction reaction involves the transfer of multiple electrons and protons. At catalytic materials such as platinum, oxygen reduction may result in the production of water via a four electron reduction process. [10] However, at carbonaceous materials the reduction process at potentials less than $-1 \mathrm{~V}$ (vs SCE) proceed no further than the two-electron reduction to the associated peroxide species. [11-13]

If adsorption processes are absent, the elementary steps of this two-electron two-proton redox process can be summarized using a `scheme-of-squares', [14-16] as shown in scheme I. In this diagram a horizontal movement corresponds to an electron transfer and a vertical shift relates to a proton transfer, a concerted proton-electron transfer process [17] may be represented by a diagonal, as for example indicated by the dashed line in scheme I. The exact mechanistic pathway taken will depend on a diversity of factors including the local solution $\mathrm{pH}$, the electrode potential, the formal potentials of the one-electron redox processes, the $p K_{a s}$ of the associated intermediates and the kinetics of each fundamental step. In the case of oxygen the scheme is somewhat simplified since a number of the intermediates likely do not exist in the solution phase. In particular the peroxide species $\mathrm{HO}_{2}^{-}$ likely has a $p K_{a}$ significantly outside of the aqueous range (i.e $>14$ ). [8] At all values of $\mathrm{pH}$ the disproportionation of the superoxide intermediate, $\mathrm{O}_{2}^{-\bullet}$, to oxygen and peroxide is highly thermodynamically favorable; however, in alkaline solutions and in the absence of a suitable catalyst superoxide is found to be relatively kinetically stable, with a reported bimolecular reaction rate of less than $0.3 \mathrm{M}^{-1} \mathrm{~s}^{-1}$ at $\mathrm{pH}$ 14. [18]

Given the significance of the oxygen reduction reaction and the common place use of carbonaceous materials as either an electrode support or as catalysts in and of themselves, $[19,20]$ the present work furthers study of the oxygen reduction mechanism at a glassy carbon electrode under neutral and alkaline conditions. Despite the insightful work of Yang and McCreery [6], who briefly mentioned possible causes for this change in voltammetry at low and high $\mathrm{pH}$, however, the exact mechanistic pathway remains unclear. It is evidenced in this paper that at both $\mathrm{pH} 7$ and 13, the rate determining step for the electrochemical reduction process is the first electron transfer resulting in the formation of the superoxide intermediate. Simulations show the kinetics of the first electron transfer process is significantly faster under the more alkaline conditions, resulting in an increase in the measured apparent transfer coefficient. This experimental observation is consistent with the 
reaction proceeding via a surface adsorbed intermediate at high $\mathrm{pH}$. It is proposed that the strength of the interaction between the electrode surface and the reduction intermediates that enables the electrochemical process to be faster (more 'electrocatalytic') under alkaline conditions. A theoretical model accounting for the presence of the adsorbed intermediate is developed, the results of which are in close agreement with the experimental data at high $\mathrm{pH}$. Note that for modelling surface sensitive electrochemical reactions it is important to recognize the presence of the adsorbed intermediate. Due to the finite number of active sites available the behavior of such systems can differ significantly from those in which the intermediates are based in the solution phase. [21] Finally, we seek to build upon these insights and aim to further illuminate the physical origins of the switch in the electrochemical behavior at $\mathrm{pH} 10$, highlighting the likely importance of the electrochemical double layer. 


\section{Experimental}

Chemicals were purchased and used directly without any further purification. These were $\mathrm{KOH}$ (Aldrich, 99+\%), $\mathrm{H}_{2} \mathrm{O}_{2}$ (Fisher, 30\%); $\mathrm{NaOH}$ (Acros Organics, 98\%), $\mathrm{CsOH} \cdot \mathrm{H}_{2} \mathrm{O}$ (Aldrich, 99+\%), $\mathrm{NaCl}$ (BDH ACS 99+\%), $\mathrm{KCl}$ (Aldrich 99+\%). Phosphate Buffer (PB) was prepared with $19 \mathrm{mM} \mathrm{NaH}_{2} \mathrm{PO}_{4}$ (Sigma, 99+\%) and $81 \mathrm{mM} \mathrm{Na}_{2} \mathrm{HPO}_{4}$ (Sigma, 99\%) giving a solution of $\mathrm{pH}$ 7.4. All solutions were prepared with deionised water of resistivity not less than $18.2 \mathrm{M} \Omega \cdot \mathrm{cm}$ at $298 \mathrm{~K}$ (Millipore UHQ, Vivendi, UK) and the $\mathrm{pH}$ measured using a Hannah pH 213 meter.

Electrochemical experiments were performed using a three-electrode configuration consisting of a glassy carbon working electrode (GCE, diameter $1.5 \mathrm{~mm}, \mathrm{CH}$ Instruments, Austin, USA ), platinum wire ( 99.99\% GoodFellow, Cambridge , UK) or graphite rod (for hydrogen peroxide reduction, Sigma-Aldrich, Dorset, UK) as counter-electrode, and a saturated calomel electrode (SCE, BAS Inc., Japan) acting as a reference electrode. Voltammetric measurements were recorded using Autolab PGSTAT20 computer-controlled Potentiostat (EcoChemie, Utrecht, The Netherlands), and the commercial software GPES®. The working electrode was polished with $1.0 \mu \mathrm{m}, 0.3 \mu \mathrm{m}$, and $0.05 \mu \mathrm{m}$ aluminium oxide (Buehler Ltd., USA) slurries on a Buehler polishing pad prior to experimentation. The polished electrode was then sonicated in deionised water for $5 \mathrm{~min}$, before placing into an electrochemical cell. Cyclic voltammetry was performed by sweeping the potential linearly from $0 \mathrm{~V}$ to $-1 \mathrm{~V}$ for most experiments, unless stated otherwise. All reported results are referenced against SCE, which is $+0.24 \mathrm{~V}$ against SHE. [8] The solution was saturated with nitrogen or oxygen for at least $20 \mathrm{~min}$ before the measurement.

The simulations were made using the commercial available software DigiSim ${ }^{\circledR}[22,23]$ or MATLAB®.

\section{Theory and Simulation}

A model for a two one-electron-transfer reaction at a macroelectrode under semi-infinite diffusion of $\mathrm{A}$ and involving an adsorbed intermediate $\mathrm{B}$, is developed, where the reaction is described by the following mechanism:

$$
\begin{aligned}
& A(\text { sol })+X(\text { ads })+e^{-}=B(\text { ads }) \\
& B(a d s)+e^{-}=C(s o l)+X(a d s)
\end{aligned}
$$


here $\mathrm{A}, \mathrm{C}$ are solution-phase species, $\mathrm{B}$ is the adsorbate on the electrode, and $\mathrm{X}$ refers to an unoccupied active site on the electrode surface. Here the mechanism has been simplified such that the adsorption/desorption of the material from the electrode is considered to occur simultaneously with the electron transfer. For an ion-transfer process i.e. the electrosorption of material to the surface, viewing the process as being concerted may well, in some cases, be a reasonable approximation. [24] As the glassy carbon electrode used in this work is large enough to avoid significant edge effects associated with the mass-transport of material to and from the electrode, in this case, linear semi-infinite diffusion is assumed. In the simulation only the concentration variation in the $z$ direction (perpendicular to the electrode surface) is taken into consideration. For the solution-phase species A and C, the variation of the concentration $c$ with the reaction time $t$ can be described by the Fick's Second Law:

$$
\frac{\partial c}{\partial t}=D \nabla^{2} c=D \frac{\partial^{2} c}{\partial z^{2}}
$$

To solve the above diffusion equation, the boundary conditions of the bulk solution $(z \rightarrow \infty)$ and the electrode surface $(z=0)$ are applied. In the bulk solution, the concentrations of $A$ and $C$ keep the initial values $c_{A}^{*}$ and $c_{C}^{*}$. On the electrode surface, assuming the two one-electrontransfer steps (1) and (2) are both elementary reactions, with the application of the Fick's First Law, the reaction rates for A, B and C can be calculated:

$$
\begin{aligned}
& -\left.D_{\mathrm{A}} \frac{\partial c_{\mathrm{A}}}{\partial z}\right|_{z=0}=-k_{\mathrm{red}, 1} c_{\mathrm{A}}(z=0) \Gamma_{\mathrm{X}}+k_{\mathrm{ox}, 1}\left(\Gamma_{\max }-\Gamma_{\mathrm{X}}\right) \\
& \frac{\partial \Gamma_{\mathrm{X}}}{\partial t}=-k_{\mathrm{red}, 1} c_{\mathrm{A}}(z=0) \Gamma_{\mathrm{X}}+k_{\mathrm{ox}, 1}\left(\Gamma_{\max }-\Gamma_{\mathrm{X}}\right)+k_{\mathrm{red}, 2}\left(\Gamma_{\max }-\Gamma_{\mathrm{X}}\right)-k_{\mathrm{ox}, 2} c_{\mathrm{C}}(z=0) \Gamma_{\mathrm{X}} \\
& -\left.D_{\mathrm{C}} \frac{\partial c_{\mathrm{C}}}{\partial z}\right|_{z=0}=k_{\mathrm{red}, 2}\left(\Gamma_{\max }-\Gamma_{\mathrm{X}}\right)-k_{\mathrm{ox}, 2} c_{\mathrm{C}}(z=0) \Gamma_{\mathrm{X}}
\end{aligned}
$$

$D_{\mathrm{A}}$ and $D_{\mathrm{C}}\left(\mathrm{m}^{2} \mathrm{~s}^{-1}\right)$ are the diffusion coefficients of $\mathrm{A}$ and C. $c_{\mathrm{A}}$ and $c_{\mathrm{c}}\left(\mathrm{mol} \mathrm{m}^{-3}\right)$ are the concentrations of $\mathrm{A}$ and C. $\Gamma_{\mathrm{X}}\left(\mathrm{mol} \mathrm{m}^{-2}\right)$ is the surface coverages of the available active sites $\mathrm{X}$ and $\Gamma_{\max }\left(\mathrm{mol} \mathrm{m}^{-2}\right)$ is the surface coverage of all the active sites available. $t$ is the reaction time. $k_{\text {red, } 1}\left(\mathrm{~m}^{3} \mathrm{~mol}^{-1} \mathrm{~s}^{-1}\right)$ and $k_{\mathrm{ox}, 1}\left(\mathrm{~s}^{-1}\right)$ are the reductive and oxidative reaction rate constants of the element reaction step (1), respectively. $k_{\mathrm{red}, 2}\left(\mathrm{~s}^{-1}\right)$ and $k_{\mathrm{ox}, 2}\left(\mathrm{~m}^{3} \mathrm{~mol}^{-1} \mathrm{~s}^{-1}\right)$ are the reductive and oxidative reaction rate constants of the element reaction step (2). The latter four parameters can be described by the Butler-Volmer equation

$$
\begin{aligned}
& k_{\mathrm{red}, 1}=k_{0,1} \exp \left(-\frac{\alpha_{1} F\left(E-E_{\mathrm{f}, 1}\right)}{R T}\right) \\
& k_{\mathrm{ox}, 1}=k_{0,1} \mathrm{c}^{o} \exp \left(\frac{\beta_{1} F\left(E-E_{\mathrm{f}, 1}\right)}{R T}\right)
\end{aligned}
$$




$$
\begin{aligned}
& k_{\mathrm{red}, 2}=k_{0,2} \mathrm{c}^{o} \exp \left(-\frac{\alpha_{2} F\left(E-E_{\mathrm{f}, 2}\right)}{R T}\right) \\
& k_{\mathrm{ox}, 2}=k_{0,2} \exp \left(\frac{\beta_{2} F\left(E-E_{\mathrm{f}, 2}\right)}{R T}\right)
\end{aligned}
$$

$k_{0,1}$ and $k_{0,2}\left(\mathrm{~m}^{3} \mathrm{~mol}^{-1} \mathrm{~s}^{-1}\right)$ are the second-order standard heterogeneous electron-transfer constants of the two elementary steps (1) and (2). [25] $c^{\circ}=1 \mathrm{~mol} \mathrm{~m}^{-3}$ is taken a reference concentration used in the simulation and is applied to balance the units between $k_{\text {red }}$ and $k_{\text {ox }}$. $F, R$ and $T$ are the Faraday constant $\left(96485 \mathrm{C} \mathrm{mol}^{-1}\right)$, the gas constant $\left(8.314 \mathrm{~J} \mathrm{~K}^{-1} \mathrm{~mol}^{-1}\right)$ and the absolute temperature $(298 \mathrm{~K})$, respectively. $E(\mathrm{~V})$ is the applied electrode potential, $E_{\mathrm{f}, 1}$ and $E_{\mathrm{f}, 2}(\mathrm{~V})$ are the formal potentials of steps (1) and (2), and thus $E-E_{\mathrm{f}}$ is the relevant overpotential of the corresponding electron-transfer step. In the Butler-Volmer theory, $\alpha$ and $\beta$ are the transfer coefficients of the electron transfer step and $\alpha+\beta=1$, and are constant as a function of the applied potential.

To simulate cyclic voltammetry, the electrode potential is varied linearly as a function of the experimental time $t$ :

$$
E=\left\{\begin{array}{c}
E_{\mathrm{ini}}+v t, \quad t \leq \frac{\left|E_{\mathrm{rev}}-E_{\mathrm{ini}}\right|}{v} \\
E_{\mathrm{rev}}-v\left(t-\frac{E_{\mathrm{rev}}-E_{\mathrm{ini}}}{v}\right), \quad t>\frac{\left|E_{\mathrm{rev}}-E_{\mathrm{ini}}\right|}{v}
\end{array}\right.
$$

where $\left[E_{\text {ini, }}, E_{\text {rev }}\right]$ define the potential window of the cyclic voltammogram and $v\left(\mathrm{~V} \mathrm{~s}^{-1}\right)$ is the scan rate.

The current at the electrode can be calculated via the concentration gradient at the electrode surface:

$$
I=F S\left(-\left.D_{\mathrm{A}} \frac{\partial c_{\mathrm{A}}}{\partial z}\right|_{z=0}+\left.D_{\mathrm{C}} \frac{\partial c_{\mathrm{C}}}{\partial z}\right|_{z=0}\right)
$$

where $S$ is the surface area of the electrode.

The resulting problem is numerically solved by means of the finite difference method, of which details can be found in the textbook. [26] The home-made simulation program is written and compiled in MATLAB R2016b. 


\section{Results and discussion}

The reduction of oxygen in an oxygen-saturated solution $\left(\left[\mathrm{O}_{2}\right]=1.24 \mathrm{mM}\right)$ [27] was studied voltametrically at a glassy carbon macro electrode at both $\mathrm{pH} 7.4$ and 13. (a solution of oxygen is attained by passing pure oxygen through a solution for 20 minutes to ensure the latter is saturated with the gas) In both cases the voltammogram was started at $0.0 \mathrm{~V}$ and swept cathodically to $-1.0 \mathrm{~V}$ vs SCE (scan rate $50 \mathrm{mV} \mathrm{s}^{-1}$ ). The voltammetric reduction of oxygen in a solution of $\mathrm{pH} 7.4$ results in a broad chemically irreversible reduction wave with a peak potential at $-0.60 \mathrm{~V}$ vs SCE (Figure 1, curve i). In contrast, although the reaction is less thermodynamically favorable at $\mathrm{pH} 13$, under highly alkaline conditions a clear reductive peak is observed at the more anodic potential of $-0.40 \mathrm{~V}$ vs SCE (Figure 1, curve ii). $[1,6]$ In both experimental cases the reduction process at the carbon interface is overall irreversible and proceeds at a high overpotential. These results are in excellent agreement with the reported literature (SI Section I, Fig. S1) [6] and indicate that the reduction of oxygen

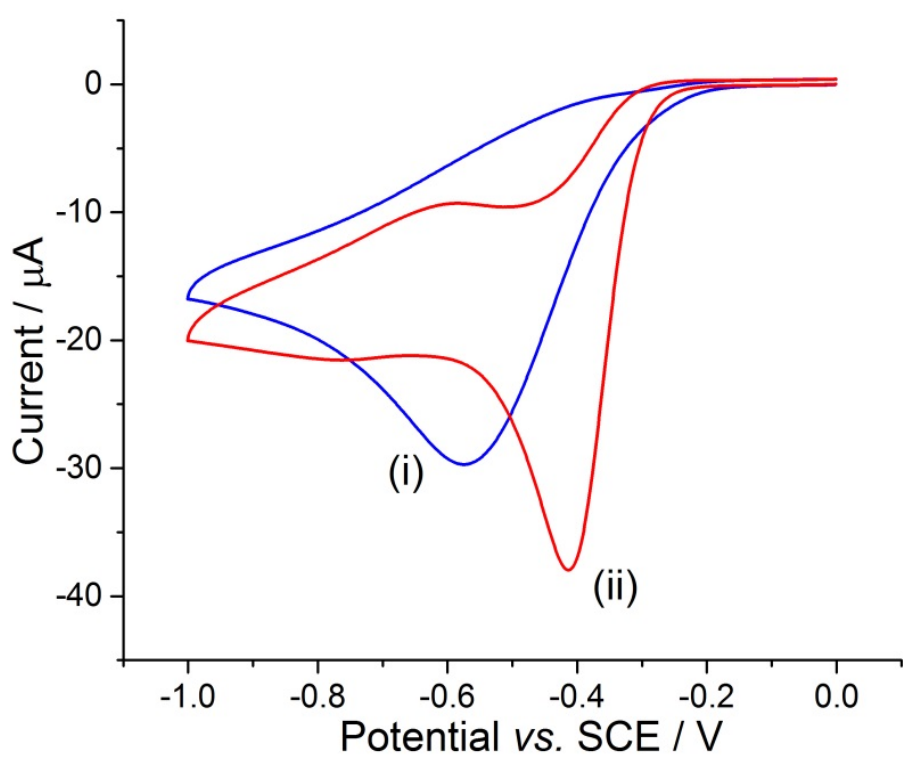

Figure 1. Oxygen reduction voltammograms at $\mathrm{pH} 7.4$ and $\mathrm{pH} 13$ at $50 \mathrm{mVs}^{-1}$ : (i) $\mathrm{pH} 7.4 ; 0.1 \mathrm{M}$ $\mathrm{KCl}, 50 \mathrm{mM}$ PB (blue line). (ii) $\mathrm{pH}$ 13; $0.1 \mathrm{M}$ $\mathrm{KOH}$ (red line)

voltammetrically occurs at lower absolute potential (as measured against a non-pH sensitive reference electrode) under conditions of high $\mathrm{pH}$. In addition, the change in the 
voltammetric wave-shape between the two $\mathrm{pHs}$ is notable. This alteration in the wave shape is best exemplified by the change in the measured transfer coefficient $(\alpha)$. Tafel analysis of the voltammograms (SI Section II, III, Fig. S2, Fig. S3) yields transfer coefficients of 0.31 $\mathbf{( \pm 0 . 0 0 2 )}$ and $0.80(\mathbf{\pm 0 . 0 0 5 )}$ at $\mathrm{pHs} 7.4$ and 13 respectively [28]. At $\mathrm{pH} 13$ the experimentally recorded Tafel slope is found to vary slightly as a function of scan rate and the measured $\alpha$ value decreases as scan rate increases, SI section III. The extracted values of the transfer coefficient are significantly less than unity; hence, for both reduction processes the rate determining step is evidenced to be the first electron transfer [29] and difference in the voltammetric response of these two systems must therefore reflect, at least in part, a change in the behavior and mechanism of this first electrochemical process. At this stage it is worth commenting that on the basis of the known solution phase acid dissociation constant $\left(p K_{a} \sim\right.$ 4.8, as shown in scheme I) [30] at both $\mathrm{pH} 7.4$ and 13 the initial product will be the nonprotonated form of the superoxide species, $\mathrm{O}_{2}^{-\bullet}$.

Within the literature one proposed mechanism for the reduction of oxygen at carbon surfaces is the mediation of the process via surface quinone functionality, under some conditions and at certain types of carbon electrodes this pathway may be operative. [3,31-34] However, as will be discussed in the present study such a surface mediated pathway is inconsistent with a number of experimental observations. We highlight that in the absence of oxygen and at an un-modified glassy carbon electrode no significant quinone redox signal is observed at either experimental $\mathrm{pH}$ in this article (see SI section IV). Moreover, due to the reported rapid homogeneous catalysis of oxygen reduction by solution phase quinones under neutral conditions, [35] in this framework it is difficult to provide a quinone based explanation for the observed shift in voltammetric behavior occur at approximately $\mathrm{pH} 10$.

\subsection{Oxygen reduction at $p H 7.4$}

In the kinetically fully irreversible limit and in accordance with 'asymmetric Marcus-Hush' theory, the experimental transfer coefficient reflects the position of the transition state between the oxidized and reduced species. [36] For an irreversible one-electron transfer process the measured transfer coefficient takes a values between 0 (reactant like) and 1 (product like), where in the case that the vibrational and solvation force constants of the 
reduced and oxidized species are equal a transfer coefficient of 0.5 is predicted. However, in the case of the reduction of oxygen to superoxide the addition of an electron to a nonbonding orbital causes a significant increase in the equilibrium bond length of the oxygen species from 1.21 to $1.33 \AA$. [37] The inner-sphere force constant of the superoxide species is hence significantly lower than found for the neutral diatomic molecule. Consequently, the electrochemical transfer coefficient for this couple is predicted to deviate from 0.5 and become closer to zero. The transfer coefficient has been previously reported to be $\sim 0.3$. $[6,38]$ Thermodynamically, the standard potential for this solution phase redox species is reportedly $-0.421 \mathrm{~V}$ (vs. SCE, $-0.18 \mathrm{~V}$ vs SHE). [9,39] Through the use of an organic film modified glassy carbon electrode McCreery [6] measured a formal potential for the electrochemical process to be $-0.416 \mathrm{~V}$ vs SCE (-0.175 V vs SHE) in $1 \mathrm{M} \mathrm{KOH}$, where the discrepancy between the two values of $-0.421 \mathrm{~V}$ and $-0.416 \mathrm{~V}$ simply reflects the altered activity coefficient of the formed superoxide species in the higher ionic strength media. 


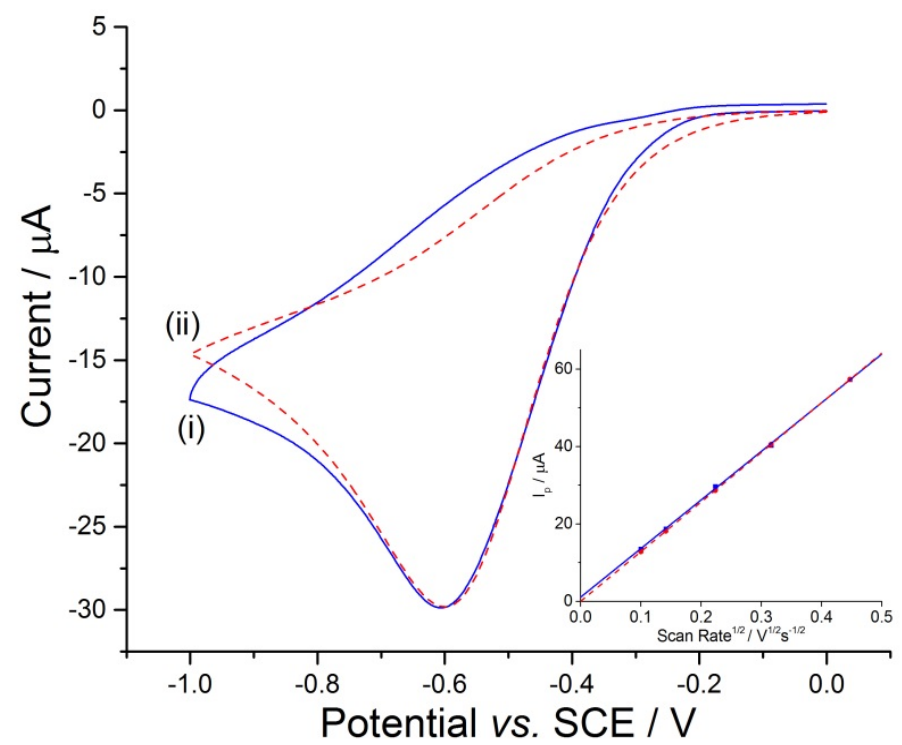

Figure 2. Comparison of voltammograms with experimental and simulated results, Cyclic voltammetry run between the range of $-1.0 \mathrm{~V}$ and $0 \mathrm{~V}$ at $50 \mathrm{mVs}^{-1}$ in $0.1 \mathrm{M} \mathrm{KCl}, 50 \mathrm{mM}$ PB: (i) Experimental (blue solid line) (ii) Simulated(red dash line) with DigiSim ${ }^{\circledR}$. Inset depicts the plot of the peak height versus the square root of scan rate for both experimental and simulated results.

The combination of the potential of the voltammetric reduction wave for oxygen at the glassy carbon electrode at $\mathrm{pH} 7.4$ and its associated low measured transfer coefficient of 0.31 ,

(SI Section II, S2.b) indicates that under these conditions the electrochemical rate determining step is the formation of the solution phase non-protonated superoxide species. After this initial rate determining electron transfer - although further reduction of the superoxide is thermodynamically favorable - the voltammetric data is insensitive to the follow-up chemical and electrochemical kinetics. However, analysis of the magnitude of the voltammetric peak current enables the total number of electron transferred to be determined.

The simulation of the electrochemical reduction ( $\mathrm{pH}$ 7.4) (SI Section V, Fig. S5) was achieved using the commercially available software DigiSim ${ }^{\circledR}$ and was modelled assuming a simple two electron transfer, where the first electron transfer was taken to be irreversible (Eirrev $\mathrm{E}$ mechanism in Testa-Reinmuth notation) [40] as described by the following reaction scheme: 


$$
\begin{aligned}
& A(s o l)+e^{-}=B(\text { sol }) \\
& B(s o l)+e^{-}=C(\text { sol })
\end{aligned}
$$

The first step represents the reduction of oxygen to superoxide (13) and second step from superoxide to hydrogen peroxide. (14) The concentration of the dissolved $O_{2}$ was set to 1.24 $\mathrm{mM}$, in accordance with previously reported values for solutions saturated with pure oxygen. [24] All species in the simulation were assumed to have a diffusion coefficient of 2.0 $\times 10^{-9} \mathrm{~m}^{2} \mathrm{~s}^{-1}$, as reported for oxygen in water at $298 \mathrm{~K}$. [41] The reduction of oxygen to superoxide has been frequently reported by previous researchers, and here the literature value of $\mathrm{E}_{0,1}=-0.421 \mathrm{~V}$ and $\alpha=0.3$ were used for the simulation. [8,38] The second electrochemical step was set to be both kinetically and thermodynamically highly driven using arbitrary values of $\mathrm{E}_{0,2}=+1.0 \mathrm{~V}$ and $\mathrm{k}_{0,2}=1.0 \times 10^{2} \mathrm{~m} \mathrm{~s}^{-1}$. Physically the second electron transfer can proceed with the additional transfer of two-protons and the exact mechanism may occur via a variety of paths including disproportionation of the superoxide, or in analogy to that found in wet DMF solution a concerted-proton-electron-transfer. [42-44] However, due to the irreversibility of the first electron transfer the voltammetry is not sensitive to the follow-up chemical kinetics or mechanism. Consequently, in modelling the system and given that the voltammetric wave shape is effectively insensitive to the kinetics of the processes after the first electron transfer (the rate determining step), the only unknown parameter here is the rate of reduction from oxygen to superoxide, $\mathrm{k}_{0,1}$. Through using the curve fitting and iterations toolkits from the DigiSim ${ }^{\circledR}$ software, a best fit with the experimental result is calculated. Figure 2. shows the best fits of the simulation model with the experimental data where a value of $8.4 \times 10^{-6} \mathrm{~m} \mathrm{~s}^{-1}$ was used for $\mathrm{k}_{0,1}$. The Mean Scaled Absolute Difference (MSAD) is calculated in order to measure the deviation of the simulations from the experimental result. The MSAD value is given by the following function:

$$
M S A D=\frac{1}{n} \sum_{i=1}^{n}\left|\frac{x_{i, \exp }-x_{i, \text { sim }}}{x_{i, \exp }}\right|
$$

It is calculated by summing the absolute percentage difference for a range of rate constants between the voltammetry of the experiment and the simulations. Simulations were carried out from $\mathrm{k}_{0,1}=6 \times 10^{-6} \mathrm{~m} \mathrm{~s}^{-1}$ up to $1.2 \times 10^{-5} \mathrm{~m} \mathrm{~s}^{-1}$, and then the MSAD value plotted against the different $\mathrm{k}_{0,1}$ used in simulation. It is confirmed that the $\mathrm{k}_{0,1}$ value which gives minimum 
error to be in the range of $8-9 \times 10^{-6} \mathrm{~m} \mathrm{~s}^{-1}$. The close fit of the simulated response at $\mathrm{k}_{0,1}=8.4$ $\times 10^{-6} \mathrm{~m} \mathrm{~s}^{-1}$ and experimental voltammetric response (Figure 2 ) clearly indicates that at $\mathrm{pH}$ 7.4 the reduction process results are consistent with the overall the two-electron reduction of oxygen to hydrogen peroxide.

In summary, the kinetic and other parameters are consistent with the following,

$$
\begin{gathered}
E_{0,1}=-0.42 \mathrm{~V} ; k_{0,1}=8.4 \times 10^{-6} \mathrm{~m} \mathrm{~s}^{-1} ; \alpha_{1}=0.3 \\
E_{0,2}=+1.0 \mathrm{~V} ; k_{0,2}=1.0 \times 10^{2} \mathrm{~ms}^{-1} ; \alpha_{2}=0.5 \\
{[A]=1.24 \mathrm{mM} ; D(A, B, C)=2.0 \times 10^{-9} \mathrm{~m}^{2} \mathrm{~s}^{-1}}
\end{gathered}
$$

\subsection{Oxygen Reduction at $\mathrm{pH} 13$}

Next we consider the mechanism of oxygen reduction at a glassy carbon electrode at $\mathrm{pH} 13$. The associated shift in the transfer coefficient from 0.30 to 0.80 either indicates an alteration in the position of the transition state associated with the reduction and/or reflects a change in the kinetics of the electrochemical reduction. Importantly, at the higher $\mathrm{pH}$ the experimental Tafel slope and hence the associated transfer coefficient is found to be sensitive to the applied scan rate. This strongly suggests the first electron transfer to be quasireversible in nature; however the second electron transfer is still completely irreversible, making the overall reaction irreversible as well. Note as the electrochemical process becomes faster the Tafel slope no longer yields the transfer coefficient as defined in the Butler-Volmer equation as both cathodic and anodic term contribute to the current. [45] For a further exploration of the analysis of the transfer coefficient of multi-step electrode processes the reader is referred to the review by Fletcher.[46] At the reversible limit the transfer coefficient as determined from the Tafel analysis reflects the number of electron being transferred. [5] Hence, the sensitivity of the experimental transfer coefficient to the experimental scan rate at high $\mathrm{pH}$ signals the quasi-reversibility of the first electron transfer. In either case the mechanism of the first electron transfer process must be altered from that found at $\mathrm{pH}$ 7.4. We infer that at high $\mathrm{pH}$ the product of the initial one-electron reduction is a surface adsorbed and not a solution phase species. The exact identity and nature of this surface adsorbed species will be returned to. However, the work first seeks to identify the number of electrons transferred to the oxygen during this voltammetric reduction process at 
high $\mathrm{pH}$. To this end the voltammetric response of oxygen reduction was studied in both potassium and sodium hydroxide at concentrations of both $0.1 \mathrm{M}$ and $1 \mathrm{M}$. For an electrochemically irreversible processes then the peak current can be quantitatively described using the irreversible form of the Randles-Sevcik equation as given by:

$$
I_{p}=2.99 \times 10^{5} n\left(n^{\prime}+\alpha\right)^{1 / 2} D^{1 / 2} A\left[O_{2}\right]_{b u l k} v^{1 / 2}
$$

$I_{p}$ is the peak current of the voltammetry, $n$ the total number of electron transferred, $n^{\prime}$ the number of the electron transfers before the rate limiting step. $\alpha$ is the reaction transfer coefficient, $\mathrm{D}$ is the diffusion coefficient of $\mathrm{O}_{2},\left[\mathrm{O}_{2}\right]_{\text {bulk }}$ is the conc. of $\mathrm{O}_{2}$ in bulk solution, A the area of the macroelectrode surface and $v$ the potential scan rate of the cyclic voltammetry.

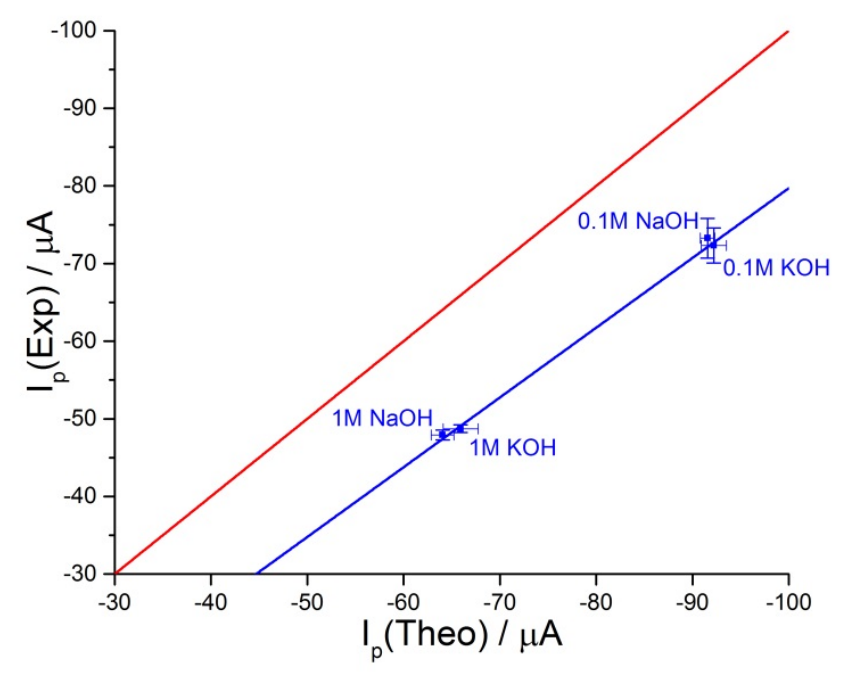

Figure 3. Scatter plot for theoretical peak current (calculated from RS equation, assuming $\mathrm{n}=2$, using $\alpha$ measured from Tafel plot, scan rate $=50 \mathrm{mV} \mathrm{s}^{-1}, \mathrm{D}=2.0 \times 10^{-9} \mathrm{~m}^{2} \mathrm{~s}^{-1}$ and $\left[\mathrm{O}_{2}\right]$ $=1.24 \mathrm{mM}$ ) against experimentally measured peak current. Linear fit for the data points (blue line), Line which experimental result equals to theoretical results (red line).

Taking the saturated oxygen concentration in solution to be $1.24 \mathrm{mM}$, [27] the literature reported diffusion coefficient in the different electrolytes [41,47] and the experimentally recorded Tafel slopes then through the irreversible form of the Randles-Sevcik equation the theoretically expected voltammetric peak current for the two electron process can be determined. Figure 3 shows a scatter plot of the experimentally determined voltammetric peak heights (as measured at a scan rate of $50 \mathrm{mV} \mathrm{s}^{-1}$ ) plotted against that predicted for an irreversible two electron reduction process. (a quasi-reversible or fully reversible process would show a higher peak current) As can be seen in all four cases the peak height is close 
to, but significantly below, that predicted theoretically for the fully irreversible two-electron transfer. Moreover, in all cases the voltammetric peak current for the reduction process is found to not be directly-proportional to the square-root of scan rate, as exemplified by plotting the voltammetric response as a function of scan rate normalized against the squareroot of scan rate (SI Section III, Fig. S3.b). This non-direct proportionality with scan rate is also observable in the inlay of Figure 4 where the non-zero intercept for the plot of peak current against the square-root of scan rate can be seen. Any proposed mechanism for the reduction of oxygen at high $\mathrm{pH}$ at the glassy carbon needs to be able to provide an explanation for the observed variability in the electrochemical response as a function of the experimental scan rate.

It should be noted that during the voltammetric reduction of oxygen at the glassy carbon surface, at these high $\mathrm{pH}$ a second reductive feature is observable at a more negative potential of ca. $-0.80 \mathrm{~V}$ vs SCE. (Fig. 4) This voltammetric feature does not arise from the further reduction of the produced hydrogen peroxide. Direct hydrogen peroxide reduction at a glassy carbon electrode is only experimentally found to occur under highly alkaline conditions ( $\mathrm{pH} 14)$ and at a more reductive potential of $-1.0 \mathrm{~V}$ vs SCE (SI Section VI, Fig. S6). The exact nature of this second reduction wave is unknown; however, simulation results indicate that it is unlikely to be due to a second parallel reduction mechanism for $\mathrm{O}_{2}$ reduction occurring at higher overpotentials (see SI section IX).

Given that the response at $\mathrm{pH} 7.4$ is consistent with the rate determining step being the formation of the solution phase superoxide species it is postulated that the product of the first electron transfer at $\mathrm{pH} 13$ is surface adsorbed in nature. The presence of this surface adsorbed species has been previously proposed by McCreery [6] who concluded that it likely has a peroxide like structure, as formed by the combination of the radical superoxide species, $\mathrm{O}_{2}^{-\bullet}$, with an available carbon radical site, $R^{\bullet}$, situated at the electrochemical interface to form $\mathrm{ROO}^{-}$. This conclusion was further corroborated by the observed change in the oxygen reduction voltammetric response at $\sim 10 \mathrm{pH}$. This change was ascribed as occurring due to the protonation of the surface adsorbed species, noting that the $p K_{a}$ of many organic peroxides are found in the range of 8.9 to 12.8 , i.e. significantly above the $p K_{a}$ value of 4.8 associated with unbound superoxide. The change around $\mathrm{pH} 10$ is possibly related to the $p K_{a}$ of the surface superoxide being comparable in this range, i.e. $p K_{a}\left(R O O^{-}\right) \sim 10$. 
$[48,49]$ Under these highly alkaline conditions the exact position of the voltammetric reduction wave is found to be sensitive to both the identity and concentration of the used electrolyte (SI section VII, Fig. S7).

On the basis of the first electron transfer resulting in the formation for a surface adsorbed superoxide species, a question arises as to the possible mechanistic routes leading to the solution phase peroxide production. First, assuming the adsorbed superoxide is bound via a

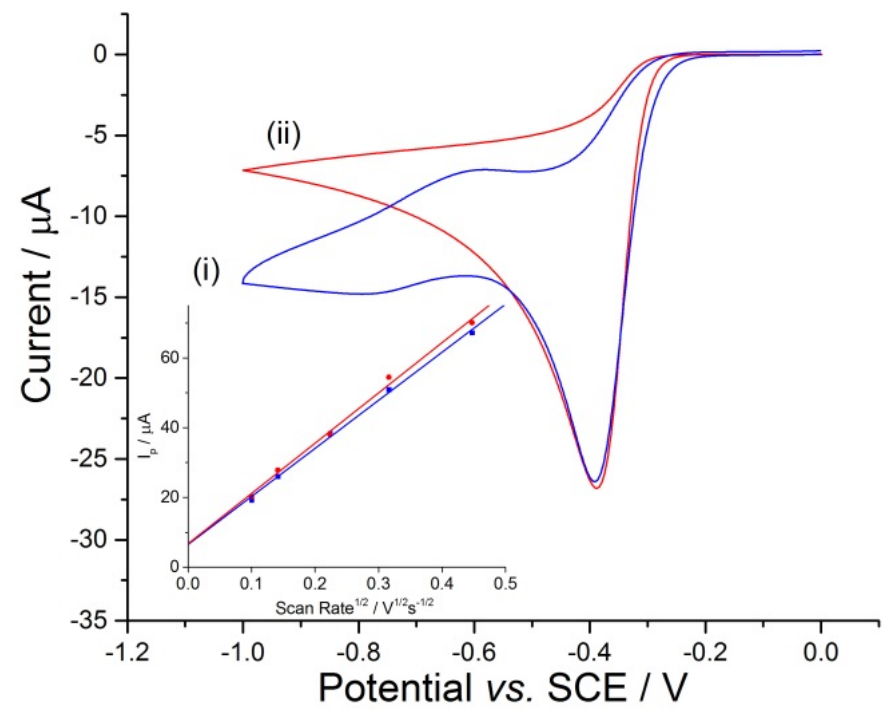

Figure 4. Comparison of voltammograms with experimental and simulated results, Cyclic Voltammetry run between the range of $-1.0 \mathrm{~V}$ and 0 $\mathrm{V}$ at $20 \mathrm{mV} \mathrm{s}^{-1}$ in $0.1 \mathrm{M} \mathrm{KOH}$ : (i) Experimental (blue line), (ii) Simulated (red line) with MATLAB ${ }^{\circledR}$. Inset depicts the plot of the peak height versus the square root of scan rate for both experimental and simulated results

carbon radical site and is hence immobile it seems unlikely that the surface reaction will proceed via a surface enabled disproportionation. [42] Second, due to the plausibly altered $\mathrm{pK}_{\mathrm{aS}}$ of the surface intermediates an ECE type mechanism for the reduction may be anticipated, however this may be discounted as it is not consistent with the observation of a different electrochemical mechanism occurring at pH 7.4. [50] Consequently, we are led to consider an EE type mechanism for the reduction of oxygen to hydrogen peroxide at the glassy carbon surface at high $\mathrm{pH}$. Taking an EE mechanism as described by the equations 1) and 2) as an illustrative and simplified model, we consider how closely this mechanism, 
which includes the presence of a surface adsorbed intermediate, can describe the observed voltammetric response.

The voltammetric response of the reduction of oxygen at $\mathrm{pH} 13$ is shown in Figure 4 . As discussed before, the non-direct proportionality of the peak current needs to be aptly described by any simulation of the experimental system.

\subsection{Simulation using adsorption mechanism for reduction at $\mathrm{pH} 13$}

The numerical simulation of the voltammetric response has been undertaken using MATLAB ${ }^{\circledR}$. In order to reduce complexity, a simple two step reduction mechanism is used for this simulation,

$$
\begin{aligned}
& A(s o l)+X(a d s)+e^{-}=B(a d s) \\
& B(a d s)+e^{-}=C(s o l)+X(a d s)
\end{aligned}
$$

The first step represents the reduction of oxygen to a surface adsorbed superoxide, consequently both the concentration of the oxygen species adjacent to the interface and the density of the active sites will influence the voltammetric response. The concentration of oxygen is taken as the saturated oxygen concentration in aqueous solution, $1.24 \mathrm{mM}$. A diffusion coefficient of $2.0 \times 10^{-9} \mathrm{~m}^{2} \mathrm{~s}^{-1}$ was used for all solution phase species. $[27,41]$ The reduction potential of oxygen to the surface bound oxygen species was assumed to be similar to the solution phase reduction potential $\mathrm{E}_{0,1}=-0.42 \mathrm{~V}$, however its value may in reality differ. (1) The second reduction potential was set to be arbitrarily high, $+0.8 \mathrm{~V}(2)$, as it is assumed to be highly thermodynamically driven. In both cases an transfer coefficient of 0.5 is assumed for $\alpha_{1}$ and $\alpha_{2}$. (2) Consequently, there are 3 unknown variables, $\mathrm{k}_{0,1}$, the rate of the first oxygen reduction and $\mathrm{k}_{0,2}$, the rate of the second reduction of surface bound superoxide and $\Gamma_{\max }$ the surface coverage of the active sites on the GC electrode. As there are no reported values for these parameters of the surface reduction of oxygen, the best approach is to estimate quantitatively. In this case, we could only estimate a fit for the experimental result.

From several iterations (SI Section VIII) by altering the unknown variables we obtain values of $\mathrm{k}_{0,1}=10 \mathrm{~m}^{3} \mathrm{~mol}^{-1} \mathrm{~s}^{-1}, \mathrm{k}_{0,2}=1 \times 10^{-8} \mathrm{~m}^{3} \mathrm{~mol}^{-1} \mathrm{~s}^{-1}, \Gamma_{\max }=1 \times 10^{-7} \mathrm{~mol} \mathrm{~m}^{-2}$. Note that the reported surface active site of a glassy carbon electrode is between $10^{-5}$ to $10^{-8} \mathrm{~mol} \mathrm{~m}^{-2}$, therefore an 
estimate of $\Gamma_{\max }=1 \times 10^{-7} \mathrm{~mol} \mathrm{~m}^{-2}$ is within a reasonable range. $[21,32,51]$

Overall, the kinetic parameters are consistent with the following,

$$
\begin{gathered}
E_{0,1}=-0.421 \mathrm{~V} ; k_{0,1}=10 \mathrm{~m}^{3} \mathrm{~mol}^{-1} \mathrm{~s}^{-1} ; \alpha_{1}=0.5 \\
E_{0,2}=+0.80 \mathrm{~V} ; k_{0,2}=1.0 \times 10^{-8} \mathrm{~m}^{3} \mathrm{~mol}^{-1} \mathrm{~s}^{-1} ; \alpha_{2}=0.5 \\
{[A]=1.24 \mathrm{mM}, D(A, B, C)=2.0 \times 10^{-9} \mathrm{~m}^{2} \mathrm{~s}^{-1}, \Gamma_{\max }(X)=1.0 \times 10^{-7} \mathrm{~mol} \mathrm{~m}^{-2}}
\end{gathered}
$$

From the inlay of Figure 4, it can be seen that this model is capable of describing the variation in the voltammetric peak height as a function of scan rate. The question arises as to how the model is capable of reproducing this alteration in the voltammetric waveshape. Initially, one might naively ascribe the variation in the peak current as a function of scan rate as resulting from a switch in the voltammetric behaviour from being overall a one-electron to a two-electron transfer, i.e. a change in the effective number of electrons transferred in the case of an ECE mechanism. [52] However, in the present case the intermediate is adsorbed and consequently cannot be lost from the surface prior to further reduction. [53] The kinetic parameters presented for the above model demonstrate the first electron transfer to be quasireversible in nature (broadly however the first electron transfer can still be viewed as being the rate determining step, as partially reflected in the transfer coefficient) and the second although assumed to be highly thermodynamically driven to be kinetically hindered. As a function of scan rate the behaviour of these two electrochemical steps will differ on the basis of their respective kinetics; the voltammetric potential of the former will be relatively insensitive to the scan rate. Conversely, the second step due to the irreversibility of the electron transfer will shift to higher over potentials at high scan rates. Consequently, over the range of experimentally studied scan rates the relative positions of these two processes differ resulting in the altered electrochemical wave shape. Further exploration of this change in the mechanism can be found in the SI section X. SI Section VIII, Fig. S8.d presents theoretical results demonstrating the alteration of the voltammetric peak as a function of the second electrochemical rate constant. [54] 
pH 7.4 in Solution

$\mathrm{O}=\mathrm{O} \stackrel{\mathrm{e}^{\ominus}}{\longrightarrow} \mathrm{O}-\mathrm{O}^{\ominus} \stackrel{\mathrm{H}^{+}}{\longrightarrow} \mathrm{HO}-\mathrm{O}^{\cdot} \stackrel{\mathrm{e}^{\ominus}}{\longrightarrow} \mathrm{HO}-\mathrm{O}^{\ominus}$

pH 13

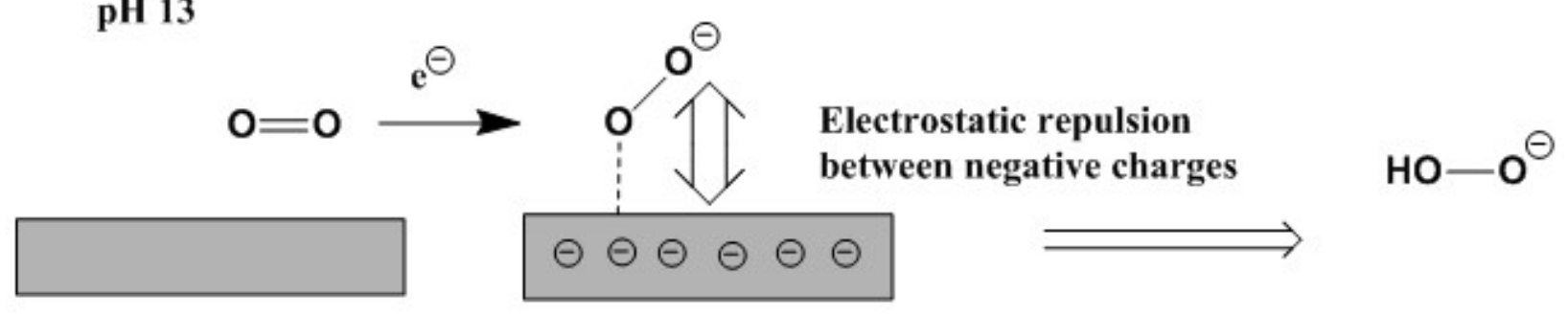

GC Electrode Surface

pH 7.4

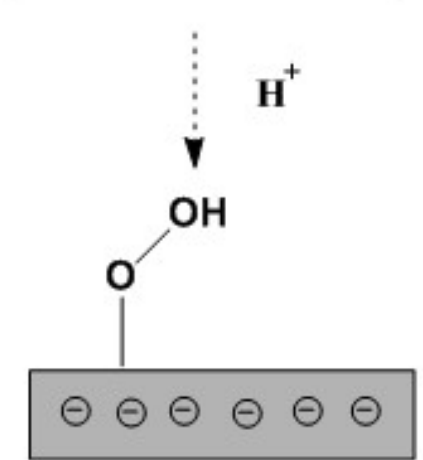

Surface peroxide could not be reduced and block the active site, inhibiting the surface pathway

Scheme II: Two mechanistic route at base and neutral conditions. The $p K_{a}$ of the surface adsorbed superoxide species is estimated from organic peroxides as being 10. [47] However in contrast, in the solution phase the $p K_{a}$ of superoxide is known to be 4.8. [8] The surface route is favored only at high $\mathrm{pH}$ due to the destabilisation of the superoxide by coulombic repulsion. The solution route is not favored because of the lack of protons. 


\subsection{Discussion of the adsorption mechanism}

Scheme II outlines the proposed mechanisms occurring at the two different solution $\mathrm{pH}$. First, at $\mathrm{pH} 7.4$ all observations are consistent with proceeding via a solution phase reaction which can be nominally modelled as an Eirrev E mechanism where the first electron transfer is rate determining and results in the formation of a solution phase superoxide species. The subsequent reduction of the superoxide is highly driven leading to the overall two-electron reduction of the oxygen to hydrogen peroxide. Conversely, under alkaline conditions $(\mathrm{pH}$ 13) the reaction is evidenced to proceed via a two-electron reduction pathway proceeding via a surface adsorbed intermediate. The non-direct proportionality of the voltammetric peak height with scan rate is consistent under this model where the second electron transfer is kinetically hindered. The irreversibility of the second electron transfer causes a shift in the relative positions of the voltammetric reduction processes as a function of scan rate. In comparing these two mechanisms an important question arises as to why the surface mediated pathway does not occur at $\mathrm{pH}$ 7.4. Previous work has argued that the protonation of the surface adsorbed intermediate inhibits the operation of this pathway. [6] However, on a purely coulombic basis one may anticipate the further reduction of the surface superoxide species to be more thermodynamically favorable in the case that it is protonated. How then may we understand this change in electrochemical mechanism? Tentatively we propose two possibilities to explain this apparent contradiction. First, we propose protonation of the surface adsorbed superoxide may alter the reduction product of the superoxide species. When situated at the surface the oxygen/oxygen bond strength may well differ from that found in the solution phase. Consequently, when the superoxide is protonated the reduction may conceivably lead to the formation of a surface bound and stable surface hydroxyl group. Hence, the formed hydroxyl groups will lead to an inactivation of the adsorption sites on the electrode surface at low $\mathrm{pH}$. Alternatively, we note that the formed superoxide species will necessarily be situated close to the electrode surface and hence may, when deprotonated, experience an electrostatic repulsion from the electrode surface [24] (noting that at these reductive potentials the electrode itself is likely to be negatively charged). The plausible importance of electrostatic repulsion with the electrode surface has recently been proposed for the inner-sphere reduction of oxygen and carbon dioxide. [55,56] This 
additional repulsive force may serve to lower the enthalpic strength of the electrode/superoxide bond. Consequently, in the absence of this additional repulsive force the protonated form may be bound strongly enough to the interface such that further reduction and release of the species from the surface is energetically unobtainable.

\section{Conclusions}

The difference in cyclic voltammetry of $\mathrm{O}_{2}$ reduction at low and high $\mathrm{pH}$ on glassy carbon electrode and macro-electrodes is evidenced to likely arise due to a change in the electrochemical mechanism. In this work numerical simulations demonstrate that the voltammetric response at $\mathrm{pH} 7.4$ is consistent with a solution phase based reaction scheme with an initial rate determining formation of the superoxide intermediate. Conversely, at high $\mathrm{pH}$ the voltammetry is consistent with a reduction mechanism involving the formation of an adsorbed intermediate. The sensitivity of the voltammetric wave-shape to the experimental scan rate is reproduced by the simulation and is explained in terms the associated shift in the relative voltammetric position of the two one-electron reduction processes as a function of the scan rate.

In combination these mechanisms illuminate the origins of the shift in peak potential for the irreversible reduction of $\mathrm{O}_{2}$ on carbon electrodes, which is counter-intuitive as the reduction occurs voltammetrically at lower potentials with high as opposed to low $\mathrm{pH}$. The adsorption of the oxygen to the active sites on the glassy carbon allows the first reduction of oxygen to superoxide to be kinetically faster (from simulation $\mathrm{k}_{0,1}=8.4 \times 10^{-6} \mathrm{~m} \mathrm{~s}^{-1}$ in solution and 10 $\mathrm{m}^{3} \mathrm{~mol}^{-1} \mathrm{~s}^{-1}$ on surface). This alteration in the kinetics of the first reduction processes is reflected in the experimentally determined transfer coefficient from 0.30 at $\mathrm{pH} 7.4$ to 0.80 at $\mathrm{pH}$ 13. The switch between the two mechanisms is proposed to occur due to the plausible protonation of the superoxide intermediate at low $\mathrm{pH}$. Two tentative explanations as to why protonation of the intermediate blocks the surface reduction mechanism are proposed. First we suggest protonation may alter the chemical product of the reduction process leading to the formation of surface peroxide groups. Second, alternatively, assuming the processes is otherwise unaltered; protonation of the surface intermediate will likely decrease the electrostatic repulsion of the intermediate from the electrode surface. This increase in the binding strength of the protonated superoxide to the electrode surface may be sufficient to 
render further reduction of the oxygen species to hydrogen peroxide energetically unfavorable.

\section{Acknowledgements}

The research leading to these results has received partial funding from the European Research Council under the European Union's Seventh Framework Programme (FP/20072013) / ERC Grant Agreement n. [320403]. 


\section{References}

[1] R.J. Taylor, A.A. Humffray, Electrochemical studies on glassy carbon electrodes, II. Oxygen reduction in solutions of low $\mathrm{pH}(\mathrm{pH}>10) \mathrm{J}$. Electroanal. Chem. Interfacial Electrochem. 64 (1975) 63-84.

[2] R.J. Taylor, A.A. Humffray, Electrochemical studies on glassy carbon electrodes: III. Oxygen reduction in solutions of low $\mathrm{pH}(\mathrm{pH}<10)$, J. Electroanal. Chem. Interfacial Electrochem. 64 (1975) 85-94.

[3] G. Jürmann, D.J. Schiffrin, K. Tammeveski, The $\mathrm{pH}$-dependence of oxygen reduction on quinone-modified glassy carbon electrodes, Electrochimica Acta. 53 (2007) 390-399.

[4] A.J. Bard, L.R. Faulkner, Electrochemical Methods: Fundamentals and Applications, $2^{\text {nd }}$ Edition. Wiley (2001).

[5] R.G. Compton, C.E. Banks, Understanding Voltammetry, $2^{\text {nd }}$ Edition. Imperial College Press (2010).

[6] H.-H. Yang, R.L. McCreery, Elucidation of the Mechanism of Dioxygen Reduction on Metal-Free Carbon Electrodes, J. Electrochem. Soc. 147 (2000) 3420-3428.

[7] J. Xu, W. Huang, R.L. McCreery, Isotope and surface preparation effects on alkaline dioxygen reduction at carbon electrodes, J. Electroanal. Chem. 410 (1996) 235-242.

[8] A.J. Bard, R. Parsons, J. Jordan, International Union of Pure and Applied Chemistry, Standard potentials in aqueous solution, CRC Press, New York (1985).

[9] W.H. Koppenol, D.M. Stanbury, P.L. Bounds, Electrode potentials of partially reduced oxygen species, from dioxygen to water, Free Radic. Biol. Med. 49 (2010) 317-322.

[10]A. Damjanovic, V. Brusic, Electrode kinetics of oxygen reduction on oxide-free platinum electrodes, Electrochimica Acta. 12 (1967) 615-628.

[11]A.J. Appleby, J. Marie, Kinetics of oxygen reduction on carbon materials in alkaline solution, Electrochimica Acta. 24 (1979) 195-202.

[12]M.S. Hossain, D. Tryk, E. Yeager, The electrochemistry of graphite and modified graphite surfaces: the reduction of O2, Electrochimica Acta. 34 (1989) 1733-1737.

[13]B. Sljukic, C.E. Banks, R.G. Compton, An overview of the electrochemical reduction of oxygen at carbon-based modified electrodes, J. Iran. Chem. Soc. 2 (2005) 1-25.

[14]J. Jacq, Schema carre: Etablissement et Discussion de L'equation Generale de la Courbe Intensite-Potentiel en Regime Stationnaire et Diffusion Convective, J. Electroanal. Chem. Interfacial Electrochem. 29 (1971) 149-180.

[15]C. Batchelor-McAuley, Q. Li, S.M. Dapin, R.G. Compton, Voltammetric Characterization of DNA Intercalators across the Full pH Range: Anthraquinone-2,6-disulfonate and Anthraquinone-2-sulfonate, J. Phys. Chem. B. 114 (2010) 4094-4100.

[16]Q. Lin, Q. Li, C. Batchelor-McAuley, R.G. Compton, Two-Electron, Two-Proton Oxidation of Catechol: Kinetics and Apparent Catalysis, J. Phys. Chem. C. 119 (2015) 1489-1495.

[17]C. Costentin, D.H. Evans, M. Robert, J.-M. Savéant, P.S. Singh, Electrochemical Approach to Concerted Proton and Electron Transfers. Reduction of the 
Water-Superoxide Ion Complex, J. Am. Chem. Soc. 127 (2005) 12490-12491.

[18]D.T. Sawyer, J.S. Valentine, How super is superoxide?, Acc. Chem. Res. 14 (1981) 393400.

[19]A.S. Arico, P. Bruce, B. Scrosati, J.M. Tarascon, W. Van Schalkwijk, Nanostructured materials for advanced energy conversion and storage devices, Nat. Mater. 4 (2005) 366377.

[20]K. Gong, F. Du, Z. Xia, M. Durstock, L. Dai, Nitrogen-Doped Carbon Nanotube Arrays with High Electrocatalytic Activity for Oxygen Reduction, Science. 323 (2009) 760-764.

[21]P. Chen, R.L. McCreery, Control of Electron Transfer Kinetics at Glassy Carbon Electrodes by Specific Surface Modification, Anal. Chem. 68 (1996) 3958-3965.

[22]A.W. Bott, S.W. Feldberg, M. Rudolph, Fitting Experimental Cyclic Voltammetry Data with Theoretical Simulations Using DigiSim ${ }^{\circledR}$ 2.1, Current Separations, $15: 2$ (1996), 6771.

[23]A.W. Bott, Simulation of Cyclic Voltammetry Using Finite Difference Methods, Current Separations, 19:2 (2000) 9-16.

[24]C. Lin, E. Laborda, C. Batchelor-McAuley, R. G. Compton, Electrical double layer effects on ion transfer reactions, Phys. Chem. Chem. Phys. 18 (2016) 9829-9837.

[25]Here it notes that the standard electron-transfer constants for steps (1) and (2) are second-order parameters involving $A \& X$ or $C \& X$ as the maximum surface coverage is treated a variable in this work. If the influence of the maximum surface coverage can be ignored, the constants can be simplified as quasi first-order constants.

[26]R.G. Compton, E. Laborda, K. R. Ward, Understanding Voltammetry: Simulation of Electrode Process. Imperial College Press (2013).

[27]R.E. Davis, G.L. Horvath, C.W. Tobias, Solubility and diffusion coefficient of oxygen in potassium hydroxide solutions, Electrochimica Acta. 12 (1967) 287.

[28] There is an important distinction between the transfer coefficient measured from a Tafel slope and the value modelled in the Butler-Volmer equation, see IUPAC publications for the case of fast electron transfer.

R. Guidelli, R.G. Compton, J.M. Feliu, E. Gileadi, J. Lipkowski, W. Schmickler, S. Trasatti, Defining the transfer coefficient in electrochemistry: An assessment (IUPAC Technical Report), Pure Appl. Chem. 86 (2014) 245-258.

R. Guidelli, R.G. Compton, J.M. Feliu, E. Gileadi, J. Lipkowski, W. Schmickler, S. Trasatti, Definition of the transfer coefficient in electrochemistry (IUPAC Recommendations 2014), Pure Appl. Chem. 86 (2014) 259-262.

See also J. Albery, Electrode Kinetics, Oxford University Press (1975).

[29] The value of the transfer coefficient and its scan rate dependency for the reduction of O2 at $\mathrm{pH} 13$ indicates that the systems kinetics are transitionary between two limits i.e. the kinetics of two processes are likely comparable in magnitude.

[30]B.H.J. Bielski, D.E. Cabelli, R.L. Arudi, Reactivity of HO2/O-2 Radicals in Aqueous Solution, J. Phys. Chem. Ref. Data. 14 (1985) 1041-1100.

[31]A. Sarapuu, K. Vaik, D.J. Schiffrin, K. Tammeveski, Electrochemical reduction of oxygen 
on anthraquinone-modified glassy carbon electrodes in alkaline solution, J. Electroanal. Chem. 541 (2003) 23-29.

[32]K. Tammeveski, K. Kontturi, R.J. Nichols, R.J. Potter, D.J. Schiffrin, Surface redox catalysis for $\mathrm{O} 2$ reduction on quinone-modified glassy carbon electrodes, J. Electroanal. Chem. 515 (2001) 101-112.

[33]K. Vaik, D.J. Schiffrin, K. Tammeveski, Electrochemical reduction of oxygen on anodically pre-treated and chemically grafted glassy carbon electrodes in alkaline solutions, Electrochem. Commun. 6 (2004) 1-5.

[34]K. Vaik, A. Sarapuu, K. Tammeveski, F. Mirkhalaf, D.J. Schiffrin, Oxygen reduction on phenanthrenequinone-modified glassy carbon electrodes in $0.1 \mathrm{M} \mathrm{KOH}$, J. Electroanal. Chem. 564 (2004) 159-166.

[35]Q. Li, C. Batchelor-McAuley, N.S. Lawrence, R.S. Hartshorne, R.G. Compton, Semiquinone Intermediates in the Two-Electron Reduction of Quinones in Aqueous Media and their Exceptionally High Reactivity towards Oxygen Reduction, ChemPhysChem. 12 (2011) 1255-1257.

[36]E. Laborda, M.C. Henstridge, C. Batchelor-McAuley, R.G. Compton, Asymmetric Marcus-Hush theory for voltammetry, Chem. Soc. Rev. 42 (2013) 4894-4905.

[37]C. Hartnig, M.T.M. Koper, Molecular dynamics simulation of the first electron transfer step in the oxygen reduction reaction, J. Electroanal. Chem. 532 (2002) 165-170.

[38]R.J. Taylor, A.A. Humffray, Electrochemical studies on glassy carbon electrodes: I. Electron transfer kinetics, J. Electroanal. Chem. Interfacial Electrochem. 42 (1973) 347354.

[39] Taking the standard state of oxygen to be unit activity and not standard pressure.

[40]V.G. Mairanovsky, On the abbreviated notation of electrode reactions, J. Electroanal. Chem. Interfacial Electrochem. 97 (1979) 103-105.

[41]K. Akita, Diffusivities of gases in aqueous electrolyte solutions, Ind. Eng. Chem. Fundam. 20 (1981) 89-94.

[42]Y. Che, M. Tsushima, F. Matsumoto, T. Okajima, K. Tokuda, T. Ohsaka, Water-Induced Disproportionation of Superoxide Ion in Aprotic Solvents, J. Phys. Chem. 100 (1996) 20134-20137.

[43]C.P. Andrieux, P. Hapiot, J.M. Savéant, Mechanism of superoxide ion disproportionation in aprotic solvents, J. Am. Chem. Soc. 109 (1987) 3768-3775.

[44]B.H.J. Bielski, A.O. Allen, Mechanism of the disproportionation of superoxide radicals, J. Phys. Chem. 81 (1977) 1048-1050.

[45]S.E. Ward Jones, F.G. Chevallier, C.A. Paddon, R.G. Compton, General Theory of Cathodic and Anodic Stripping Voltammetry at Solid Electrodes: Mathematical Modeling and Numerical Simulations, Anal. Chem. 79 (2007) 4110-4119.

[46]S. Fletcher, Tafel slopes from first principles, J. Solid State Electrochem. 13 (2009) 537549.

[47]C. Zhang, F.-R.F. Fan, A.J. Bard, Electrochemistry of Oxygen in Concentrated $\mathrm{NaOH}$ Solutions: Solubility, Diffusion Coefficients, and Superoxide Formation, J. Am. Chem. 
Soc. 131 (2009) 177-181.

[48]T.N. Das, T. Dhanasekaran, Z.B. Alfassi, P. Neta, Reduction Potential of the tertButylperoxyl Radical in Aqueous Solutions, J. Phys. Chem. A. 102 (1998) 280-284.

[49]N.A. Lange, J.A. Dean, Lange's Handbook of Chemistry, Tenth Edition, McGraw-Hill (1973).

[50]Here the chemical "C" step would be likely to correspond to the protonation of the adsorbed intermediate, this protonation step is, as will be discussed, the likely origin of the inhibition of the surface reduction route.

[51]I.-F. Hu, D.H. Karweik, T. Kuwana, Activation and deactivation of glassy carbon electrodes, J. Electroanal. Chem. Interfacial Electrochem. 188 (1985) 59-72.

[52]R.A. Marcus, On the Theory of Oxidation-Reduction Reactions Involving Electron Transfer. I, J. Chem. Phys. 24 (1956) 966-978.

[53] We comment that a mechanism in which succeeding step after formation of the adsorbed superoxide is its slow desorption from the surface, followed by electron transfer in the solution phase. Due to the second electron transfer in the solution phase being highly driven, as evidenced by the data at $\mathrm{pH} 7.4$ such a mechanism is incapable of explaining this non-direct proportionality of the peak height with the square root of scan rate.

[54]An analogous situation is found for the oxidation of the guanine at carbon surfaces, this example differs in that the intermediate in this case is in the solution phase and is not adsorbed.

[55]J.K. Nørskov, J. Rossmeisl, A. Logadottir, L. Lindqvist, J.R. Kitchin, T. Bligaard, H. Jónsson, Origin of the Overpotential for Oxygen Reduction at a Fuel-Cell Cathode, J. Phys. Chem. B. 108 (2004) 17886-17892.

[56]J.H. Montoya, C. Shi, K. Chan, J.K. Nørskov, Theoretical Insights into a CO Dimerization Mechanism in CO2 Electroreduction, J. Phys. Chem. Lett. 6 (2015) 2032-2037. 